\title{
DIFFERENTIAL EXPRESSION OF CD150/SLAMF1 IN NORMAL AND MALIGNANT B CELLS ON THE DIFFERENT STAGES OF MATURATION
}

\author{
I.M. Gordiienko, L.M. Shlapatska, L.M. Kovalevska, S.P. Sidorenko* \\ Department of Molecular and Cell Pathobiology, R.E. Kavetsky Institute of Experimental Pathology, \\ Oncology and Radiobiology, NAS of Ukraine, Kyiv 03022, Ukraine
}

\begin{abstract}
Background: Within B-cell lineage cell surface receptor CD150/SLAMF1 is broadly expressed starting from pre-B cells with upregulation toward plasma cells. However, expression of CD150 is rather limited on the surface of malignant B cells with the block of differentiation at the different stages of maturation. The aim of our work was to explore CD150 expression both on protein and mRNA levels with the emphasis on CD150 isoforms in malignant B-cell lines at the different stages of maturation in comparison with their normal B cell counterparts. Materials and Methods: Studies were performed on normal tonsillar B-cell subpopulations, Blymphoblastoid cell lines, malignant B-cell lines of different origin, including pre-B acute lymphoblastic leukemia, Burkitt's lymphoma, Hodgkin's lymphoma, and multiple myeloma. Protein CD150 expression was assessed by western blot analysis and the expression level of CD150 isoforms was evaluated using qRT-PCR. Results: Despite the similar CD150 expression both on mRNA and protein levels in normal B-cell subsets and B-lymphoblastoid cell lines, malignant B-cell lines demonstrated substantial heterogeneity in CD150 expression. Only Hodgkin's lymphoma cell lines, Burkitt's lymphoma cell lines BJAB and Raji, and also pre-B cell line BLIN-1 expressed CD150 protein. At the same time total CD150 and mCD150 mRNA was detected in all studied cell lines excluding pre-B cell line REH. The minor SCD150 isoform was found only in Hodgkin's lymphoma cell lines and Burkitt's lymphoma cell line Raji. The nCD150 isoform was broadly expressed in tested B cell lines with exception of REH and Daudi. Conclusion: Malignant Bcell lines at the different stages of maturation only partially resemble their normal counterparts by CD150 expression. In malignant B-cell lines, CD150 expression on mRNA level is much broader than on protein level. CD150 isoforms are differentially expressed in normal and malignant $B$ cells with predominant expression of $\mathrm{mCD} 150$ isoform.
\end{abstract}

Key Words: CD150/SLAMF1, CD150 isoforms, B-cell subsets, B-cell lines.

CD150 (IPO3/SLAM) is multifunctional type I transmembrane glycoprotein that belongs to signaling lymphocytic activation molecule (SLAM) family within the immunoglobulin superfamily of surface receptors [1-3]. It is expressed on activated T and B cells, activated monocytes, macrophages and dendritic cells [4-7]. Within B-cell lineage there is a noticeable heterogeneity in CD150 cell surface expression. Nearly half of B-cell progenitors in bone marrow (pro-B and pre-B cells) express CD150 on the cell surface [8]. The level of CD150 surface expression is dropped on immature $B$ cells and increased from the stage of naive $B$ cells toward plasma cells [8-10]. At the same time, expression of CD150 is rather limited on the surface of malignant $B$ cells with the block of differentiation at the different stages of maturation. High level of CD150 surface expression is observed in hairy cell leukemia, classical Hodgkin's lymphoma (HL), subtype of diffuse large $B$-cell lymphoma with activated $B$ cell phenotype (ABCDLBCL), and primary cutaneous follicular centre B-cell lymphoma [11-13]. Chronic lymphocytic leukemia is characterized by heterogeneity of CD150 expression with higher surface CD150 expression in mutated

Submitted: April 27, 2016.

*Correspondence: $\quad$ Fax: +380442581656 ;

E-mail: svitasyd@yahoo.com

Abbreviation used: B-LCL - B-lymphoblastoid cell line; $\mathrm{BL}$ Burkitt's lymphoma; EBV - Epstein - Barr virus; HL - Hodgkin's lymphoma; $\mathrm{MM}$ - multiple myeloma; pre-B ALL - pre-B acute lymphoblastic leukemia; qRT-PCR - quantitative reverse transcription polymerase chain reaction; SLAM - signaling lymphocytic activation molecule.
CLL subgroup [14, 15]. It should be emphasized that in the part of CLL cases CD150 expression was lost during malignant transformation [15]. The low level of CD150 expression was also observed in mantle cell lymphoma [12]. However, DLBCL with germinal centre phenotype, small lymphocytic lymphoma, lymphoplasmacytic lymphoma, Burkitt's lymphoma (BL), pre-B acute lymphoblastic leukemia (pre-B ALL), and multiple myeloma (MM) were CD150 negative [11-13]. The differential CD150 cell surface expression in B-cell malignancies could be explained by several mechanisms: aberrant translocation of CD150 to the plasma membrane, disruptions in regulation of CD150 expression on the transcriptional and/or translational levels.

CD150 is encoded by SLAMF1 gene that is localised on the long arm of chromosome 1 at bands 1q21-24 within SLAM family gene cluster [1-3]. Several alternatively spliced isoforms of CD150 have been identified [5, 16, 17] (Fig . 1). Canonical transmembrane CD150 isoform ( $m C D 150$ ) with two ITSM signaling motifs in cytoplasmic domain is the most widely studied isoform. All available data about CD150 mediated signaling is concerning mCD150 isoform [1]. Expression of secreted CD150 isoform (SCD150), which lacks transmembrane region, was detected in activated normal $B$ and T cells, mature dendritic cells, cases of classical $\mathrm{HL}$ and $\mathrm{HL}$ cell lines, in blood serum of healthy individuals and patients with rheumatoid arthritis [6, $16,18-20]$. Since CD150 is a self-ligand, SCD150 isoform may interact with transmembrane CD150 in local microenvironment and initiate signaling pathways that 
leads to regulation of $\mathrm{CD} 150^{+}$cells function and fate. In addition, variable membrane CD150 (vmCD150) isoform with truncated cytoplasmic tail and cytoplasmic CD150 (cCD150) isoform lacking leader sequence were predicted [5]. Recently, a novel spliced isoform of CD150 (nCD150) with alternatively cytoplasmic tail was identified [17]. The nCD150 is characterized by $83 \mathrm{bp}$ insert after transmembrane region. Cytoplasmic tail of nCD150 lacks ITSM motifs and its signaling properties are still unknown [17].

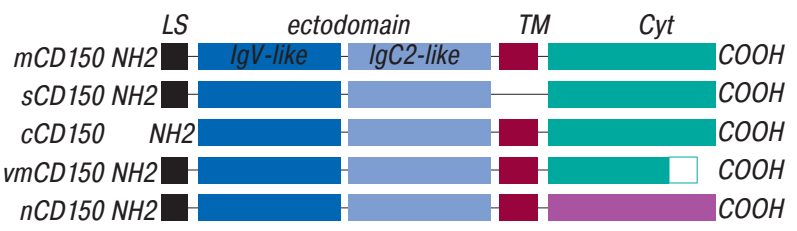

Leader sequence

Extracellular lgV-like - domain

Extracellular IgC2-like - domain

Transmembrane domain

Cytoplasmic tail with 2 ITSM

Truncated cytoplasmic tail with 1 ITSM

Alternative cytoplasmic tail without any known signaling motifs

Fig. 1. Structure of CD150 isoforms. mCD150 isoform - canonical transmembrane form of CD150 with two ITSM motifs in cytoplasmic tail. Soluble or secreted form of CD150 (sCD150) lacks transmembrane domain. Cytoplasmic CD150 isoform (CCD150) is devoid of the leader sequence, and variable CD150 isoform (vmVD150) has one ITSM motif and truncated cytoplasmic tail. The novel CD150 ( $\mathrm{nCD} 150$ ) isoform due to the additional insert after transmembrane region possess the alternative cytoplasmic tail without any known signaling motifs

The existence of alternatively spliced CD150 isoforms with different signaling properties indicates that they may mediate multiple functions by differential expression in lymphoid population at different stages of cell maturation. Up to date, there is no complete information about differential expression of CD150 isoforms in malignant $B$ cells and the contribution of different isoforms to CD150-mediated signaling. In this study, we are focused on evaluation of general CD150 protein level and mRNA expression levels of CD150 splice isoforms with conventional cytoplasmic tail (mCD150), alternatively spliced cytoplasmic tail (nCD150) and soluble secreted CD150 (sCD150) in malignant B-cell lines at the different stages of differentiation in comparison with their normal B-cell counterparts. To assess the expression level of CD150 isoforms we performed quantitative reverse transcription polymerase chain reaction (qRT$\mathrm{PCR}$ ) using primers that recognize unique regions of CD150 transcripts for each of isoforms. Up to now, qRT-PCR is the only method that allows evaluating differential expression of CD150 isoforms, since all known antibody recognised extracellular part of CD150, which is identical in all CD150 isoforms.

\section{MATERIALS AND METHODS}

Isolation of normal B cells subpopulations. Tonsils were obtained from patients undergoing tonsillectomy. Mononuclear cell populations were isolated by Lymphoprep (Axis-Shield PoCAS, Norway) density gradient centrifugation. The obtained cells were then depleted of T cells by E-rosetting with previously prepared sheep red blood cells. Subpopulations of normal $B$ cells were isolated from total tonsillar B cells using direct magnetic separation. Dynabeads coated with sheep anti-mouse IgG (Dynal Biotech ASA, Norway) were washed twice in PBS+2\% FCS and incubated either with mouse anti-CD38 $(10 \mathrm{\mu g} / \mathrm{ml})$ (Immunotech, France), or anti-CD27 (10 $\mu \mathrm{g} / \mathrm{ml})(B D$ Biosciences Pharmingen, USA), or anti-IgD (10 $\mu \mathrm{g} / \mathrm{ml}$ ) (Immunotech, France) mAb during 40 min at $4{ }^{\circ} \mathrm{C}$ with rotation. Then, CD38-beads, CD27-beads and lgD-beads were washed 3 times with cold PBS $+2 \%$ FCS and added to suspension of total tonsillar B cells at concentration of four beads per cell with next incubation during 30 min at $4{ }^{\circ} \mathrm{C}$ with rotation. Using combination of depletion and positive isolation, the following subpopulations of tonsillar $\mathrm{B}$ cells were isolated: $\operatorname{lgD}^{+} \mathrm{CD}^{-} 7^{-} \mathrm{CD} 38^{-}$ (naïve B cells), $\mathrm{CD}^{2} 8^{+} \mathrm{CD}^{-} 7^{-} \mathrm{lgD} \mathrm{D}^{-}$(germinal centre $B$ cells) - CD27 ${ }^{+}$CD38-lgD ${ }^{-}$(memory B cells).

Cell lines. The B-lymphoblastoid cell lines (B-LCL) T5-1, MP-1, CESS, 6.16; pre-B ALL cell lines REH, BLIN-1, NALM6; BL B-cell lines Ramos, Raji, BJAB, Daudi; HL B-cell lines KM-H2, L428, and MM cell line RPMI 8226 were kindly provided by Prof. Edward Clark (University of Washington, Seattle, WA, USA). HL cell line L1236 from DSMZ was kindly provided by Prof. Eva Klein (Karolinska Institute, Stockholm, Sweden). Cell lines were maintained in RPMI 1640 medium supplemented with $10 \%$ FCS, 2 mM L-glutamine and antibiotics at $37{ }^{\circ} \mathrm{C}$ in a humidified atmosphere containing $5 \% \mathrm{CO}_{2}$.

Biochemical methods. Cell pellets were lysed in Triton lysis buffer ( $150 \mathrm{mM} \mathrm{NaCl}, 1 \mathrm{mM}$ EDTA, $1 \mathrm{mM}$ EGTA, $20 \mathrm{mM}$ Tris pH = 8.0, 1\% Triton X100) containing cocktails of protease and phosphatase inhibitors (Sigma, USA). Lysates were subjected to sodium dodecyl sulfate polyacrylamide gel electrophoresis. Proteins were transferred to Immobilon-NC membranes (Millipore, USA) and blocked during $5 \mathrm{~h}$ with $5 \%$ non fat dried milk in Tris-buffered saline with Tween 20 (TBS-T). Membranes were incubated with primary anti-CD150 rabbit antibodies (Sino Biologicals Inc., China) at $4{ }^{\circ} \mathrm{C}$ overnight followed by secondary goat anti-rabbit HRP-conjugated antibodies (Santa Cruz Biotechnology, USA) for $2 \mathrm{~h}$ at room temperature. Clarity Western ECL substrate (Immuno-Star HRP, BioRad, USA) and medical X-ray film (AGFA, Belgium) were used for visualization of immunoreactions.

$\boldsymbol{q} \boldsymbol{R} \boldsymbol{T}$-PCR. Total RNA isolation, cDNA synthesis and real-time PCR were performed as described earlier [21]. The following primers for real-time PCR were used: ExtrCD150 (isoforms containing extracellular part of CD150 receptor): For 5'-AGGCCCTCCACGTTATCTA-3', Rev 5'-GCAAAAGCGCTGAACTGA-3'; Cyt-n CD150 (isoform containing alternative cytoplasmic tail): For 5'-TGAGAAGAAGAGCCACCTTGA-3', Rev 5'- GGTTCGTTTTACCATGGGAAG-3'; Cyt-m CD150 (isoforms containing conventional cytoplasmic tail): For 5'-GTGTATGCTGGGCTGTTAGG-3', Rev 5'-AGAGGTAAAACGAACCATTACCA-3'; 
soluble, secreted CD150: For 5'-AGACCCCTCAGGTAAAACG-3'; Rev 5'-TCTGGACTTGGGCATAGATCG-3'. As internal control gene for normalization was chosen TATA-box binding protein (TBP): For 5'-CCACTCACAGACTCTCACAAC-3'; Rev 5'-CTGCGGTACAATCCCAGAACT-3'. The PCR cycling conditions were the following: $10 \mathrm{~min}$ at $95^{\circ} \mathrm{C}, 40$ cycles of $15 \mathrm{~s}$ at $95^{\circ} \mathrm{C}$ and $40 \mathrm{~s}$ at $62^{\circ} \mathrm{C}$ for sCD 150 and $10 \mathrm{~min}$ at $95^{\circ} \mathrm{C}, 40$ cycles of $15 \mathrm{~s}$ at $95^{\circ} \mathrm{C}$ and $40 \mathrm{~s}$ at $64^{\circ} \mathrm{C}$ for ExtrCD150, cyto-mCD150 and cyto-nCD150. Applied Biosystems 7500 system software was used for data analysis. Ct values were determined for the internal control (TBP) and the tested isoforms at the same threshold level in the exponential phase of the PCR amplification curves. The threshold cycle (CT) method was used to calculate expression level relative to the endogenous control. Results were presented in relative units (r.u.). Dissociation curve analysis was performed after every run to check the specificity of the reaction. For all type of cells, $3-5$ reactions (each in triplicates) were run for each isoform with internal control and standard error of the mean (SEM) was calculated.

\section{RESULTS AND DISCUSSION}

CD150 expression levels were mostly studied using flow cytometry on live cells detecting its cell surface expression [4, 8, 14, 15]. To evaluate expression of all CD150 isoforms on protein level we used western blot analysis. CD150 was expressed at approximately the same level in tonsillar normal B-cell subpopulations with slightly higher level in germinal centre B cells. The lowest level of CD150 was detected in memory B cells (Fig. 2, I). B-LCL (T5-1, MP-1, 6.16, and CESS) and $\mathrm{HL}$ cell lines (KM-H2, L428 and L1236) were characterized by high CD150 expression level that exceeds its in normal B cells up to four times (Fig. 2, II, V and data not shown). The germinal centre B cells are the developmentally related normal lymphoid counterparts of $B L B$ cells [22, 23], but not all BL cell lines expressed CD150 on the cell surface $[4,11,13]$. CD150 protein expression was detected at the comparatively same level with germinal centre B cells only in BJAB and Raji but not in Ramos and Daudi BL cell lines (Fig. 2, IV). MM derives from plasma cells [24] that have the highest level of CD150 surface expression [8-10]. However, MM cell line RPMI-8226 did not express CD150 protein (Fig. 2, VI). Only one pre-B ALL cell line (BLIN-1) was CD150 positive (Fig. 2, III), despite CD150 expression was detected on the cell surface of nearly half of normal pre-B cells [8]. Taken together, our data suggest that malignant $B$ cells are characterized by aberrant expression of CD150 on protein level, in comparison with their normal B cell counterparts.

To answer the question whether CD150 is transcribed in CD150 negative B-cell lines and which of the CD150 isoforms are expressed in malignant B-cell lines we performed qRT-PCR using primers that specifically recognize different $C D 150$ domains.

Primers that are specific to extracellular domain of CD150 (extrCD150) allow to evaluate the general
mRNA expression level of all CD150 isoforms. Primers to conventional cytoplasmic tail recognise the major transmembrane mCD150 isoform (mCD150) and also minor cytoplasmic CCD150 as well as truncated vmCD150 isoforms. Using specific primers to alternative insert in cytoplasmic tail we were able to detect expression of a novel nCD150. For the detection of secreted, soluble SCD150 isoform we used primers that allow excluding the transmembrane domain.

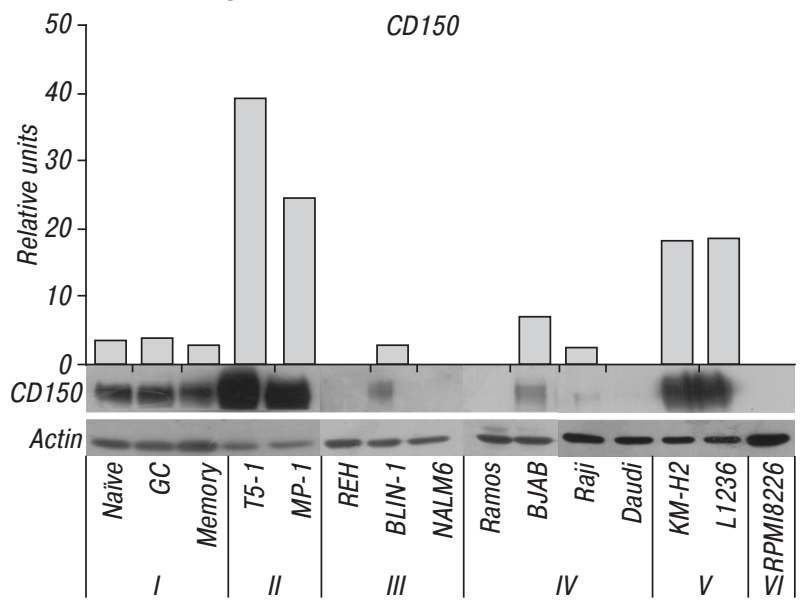

Fig. 2. The expression level of $C D 150$ protein in subpopulation of normal tonsillar B cells (I), B-LCL (II), pre-B-ALL cell lines (III), $\mathrm{BL}$ cell lines (IV), HL cell lines (V), MM cell line (VI). Western blot analysis. Actin expression level was used as loading control. The level of CD150 expression was normalized against the level of actin using Adobe Photoshop CS5 program and presented as relative units

CD150 mRNA (as indicated by extCD150) was detected in all tested normal B-cell subsets (Fig. 3, a). The mCD150 isoform was expressed at slightly lower level than the general CD150 mRNA (Fig. 3, b). The sCD150 mRNA expression was hundreds of times lower than general CD150 mRNA level in total and germinal centre B cells. At the same time, SCD150 isoform was practically undetectable in naïve and memory B-cell subsets (Fig. 3, C). The expression level of $\mathrm{nCD} 150$ isoform was tenfold lesser, in comparison with the expression of total CD150 or mCD150. It should be noticed that maximum nCD150 mRNA level was detected in memory B cells (Fig. $3, d$ ). Thus, the mCD150 transmembrane isoform with conventional cytoplasmic tail is a predominant CD150 isoform in subpopulations of normal tonsillar B cells.

Total CD150 (extCD150) mRNA was expressed at the highest level in B-LCL: up to $19.03 \pm 2.3$ r.u. in T5-1 B-LCL (Fig. 4, a I). All B-LCLs used in our study are Epstein - Barr virus (EBV) immortalized normal $B$ cells with established type III EBV latency. It was previously shown that infection of B cells by EBV upregulated cell surface expression of CD150. Moreover, expression of CD150 depends on EBV-encoded oncogene LMP1 that is highly expressed in type III EBV latency [25]. That is why elevated CD150 mRNA and protein expression level in B-LCL, compared to normal $B$ cells, could be EBV-dependent. The total CD150 (extCD150) mRNA was detected also in all tested $\mathrm{HL}$ cell lines and two BL cell lines (BJAB and Raji) that correlated with 
CD150 protein expression level and did not exceeded such in normal B cells. Moreover, it was expressed at the low level (up to 0.1 r.u.) in cell lines NALM6, Ramos, Daudi, and RPMI 8226 (Fig. 4, a), which did not express CD150 on protein level (see Fig. 2).
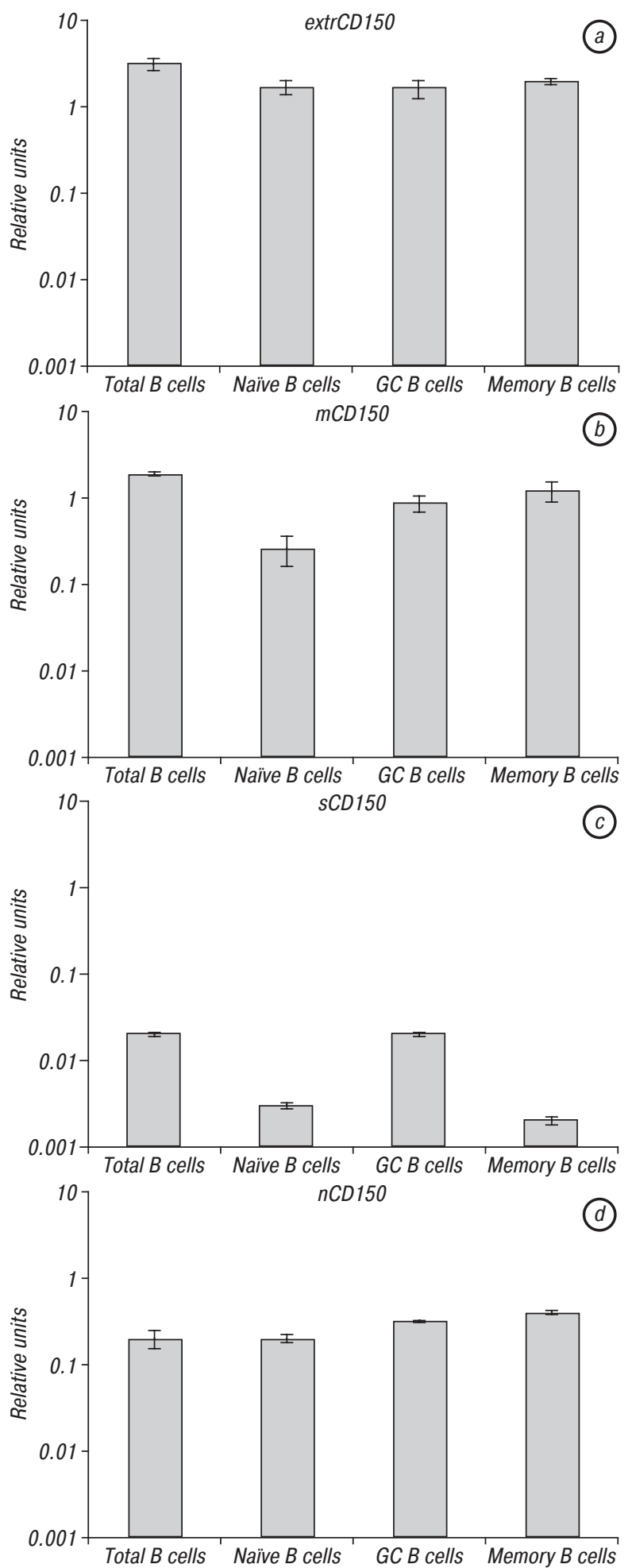

Fig. 3. The mRNA expression level of total CD150 isoforms extrCD150 (a), mCD150 isoform (b), sCD150 isoform (c), and nCD150 $(d)$ in total normal B cells, naïve B cells, germinal centre $B$ cells and memory B cells. Real-time qRT-PCR. The results, presented as mean of triplicates ( \pm SEM), are from one of three independent experiments

In malignant B-cell lines expression level of mCD150 transmembrane isoform with conventional cytoplasmic tail repeated overall expression level for total CD150 (Fig. 4, b). It was shown that sCD150 isoform was expressed in all tested B-LCL, HL cell lines and BL cell line Raji. At the same time, sCD150 was not found in all tested pre-B ALL cell lines, BL cell lines Ramos, BJAB and Daudi, and MM cell line RPMI 8226 (Fig. 4, c, II, III, V).
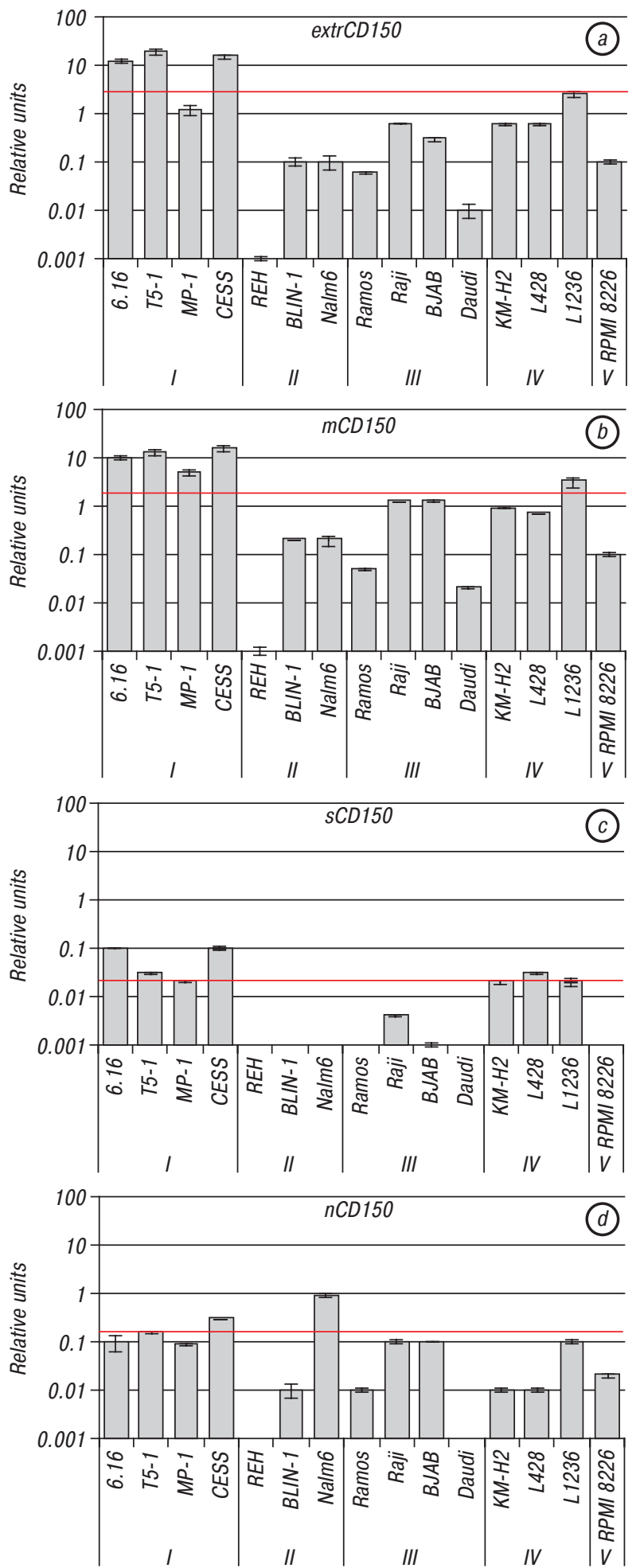

Fig. 4. mRNA expression level of total CD150 isoforms extrCD150 (a), $\mathrm{mCD} 150$ isoform (b), sCD150 isoform (c), and nCD150 (d) in B-LCLs (I), pre-B ALL cell lines (II), BL cell lines (III), HL cell lines (IV), MM cell line (V). Real-time qRT-PCR. The results presented as mean of triplicates ( \pm SEM) are from one of three independent experiments. Red lines show the expression level of corresponding CD150 isoforms in total tonsillar B cells 
On the other hand, the elevated over normal B cells level of SCD150 isoform was observed in 6.16 and CESS B-LCLs (Fig. 4, $d$, I). The nCD150 mRNA was revealed in the majority of tested cell lines with the exception of pre-B ALL cell line REH and BL cell line Daudi (Fig. 4, d). Similarly to normal B-cell subsets, in malignant Bcell lines expression level of nCD150 was lower, compared to the expression of $\mathrm{mCD} 150$ isoform (Fig. 4, d). It was surprising that in pre-B-ALL cell line NALM-6 the nCD150 was a major CD150 isoform (Fig. 4, d, II). Our results demonstrated that CD150 isoforms are differentially expressed in studied B-cell lines with predominant expression of $\mathrm{mCD} 150$ transmembrane splice isoform with conventional cytoplasmic tail.

90-95\% of human lymphoma/leukemia in adults are of B cell origin. CD150 is expressed at different stages of $B$ cell maturation from pro-B to plasma cells with upregulation of its expression during differentiation $[8,9]$. However, CD150 cell surface expression is rather limited on malignant $B$ cells $[12,13,20]$. In our study, we revealed heterogeneity of CD150 expression in malignant B-cell lines (summarized in Table) that may reflect the different stages of differentiation of normal counterparts of malignant cells.

Table. Expression of CD150 on the protein and mRNA level in normal $B$-cell subsets and B-cell lines

\begin{tabular}{|c|c|c|c|c|c|c|}
\hline \multirow[b]{2}{*}{$\begin{array}{l}\text { Subpopulation } \\
\text { of normal B cells } \\
\text { and cell lines }\end{array}$} & \multirow[b]{2}{*}{$\begin{array}{l}\text { Cell surface } \\
\text { expression }\end{array}$} & \multirow{2}{*}{$\begin{array}{l}\text { General ex- } \\
\text { pression level } \\
\text { (Western blot } \\
\text { analysis) }\end{array}$} & \multicolumn{4}{|c|}{ mRNA expression } \\
\hline & & & 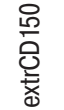 & 음 & 용 & 윰 \\
\hline Naïve B cells & $+[9]$ & + & +++ & ++ & - & ++ \\
\hline GC B & $+[9]$ & + & +++ & +++ & + & ++ \\
\hline cells & $+[9]$ & + & ++ & +++ & - & +++ \\
\hline $6.16 \mathrm{EBV}+$ & $+++[4]$ & ++ & +++ & +++ & + & + \\
\hline & $+++[34]$ & +++ & +++ & +++ & + & ++ \\
\hline MP-1 EBV+ & $+++[34]$ & +++ & +++ & +++ & + & + \\
\hline CESS EBV+ & $+++[4]$ & ++ & +++ & +++ & + & ++ \\
\hline REH EE & $-[4]$ & - & - & - & - & - \\
\hline BLIN-1 EBV- & $\begin{array}{c}++ \\
\text { (unpubl.) }\end{array}$ & + & ++ & + & - & + \\
\hline Nalm & $-[4]$ & - & ++ & + & - & ++ \\
\hline Ramo & $-[4]$ & - & + & + & - & + \\
\hline Raji E & $++[4]$ & + & ++ & + & + & + \\
\hline BJAB & $++[4]$ & + & ++ & + & - & + \\
\hline Daudi EBV+ & $-[8]$ & - & + & + & - & - \\
\hline KM-H2 EBV- & $+++[20,35]$ & ++ & ++ & ++ & + & + \\
\hline L428 EBV- & $+++[20,35]$ & ++ & ++ & + & + & + \\
\hline L1236 EBV- & $+++[20,35]$ & ++ & +++ & +++ & + & ++ \\
\hline RPMI 8226 EBV- & $-[4]$ & - & ++ & ++ & - & + \\
\hline
\end{tabular}

Note: +++ high expression level; ++ medium expression level; + low expression level; - expression was not detected.

It could be true for pre-B-cell lines, since about half of normal pro-B and pre-B cells express CD150. Only in one pre-B-cell line REH CD150 was absent both on mRNA and protein level. In NALM6 CD150 was expressed only on mRNA level, and in BLIN-1 CD150 protein was also detected. Probably, these pre-B-cell lines represent their normal counterparts at the sequential stages of differentiation. As to BL cell lines, the situation is different. Two of four $B L$ cell lines express CD150 protein independently of EBV presence. However, all tested BL cell lines express CD150 mRNA that did not exceed the level in normal $B$ cells. At the same time, germinal centre B cells express CD150 both on mRNA and protein level. Contrary to BL cell lines, in studied HL cell lines, which derived from crippled, pre-apoptotic germinal centre B cells, we found the correlation between CD150 expression on mRNA and protein levels. Despite the high level of CD150 expression on the surface of normal plasma cells, MM cell line RPMI 8226 expressed CD150 only on mRNA level. Thus, we found that in malignant $B$ cell lines CD150 expression on mRNA level is much broader than on protein level.

$95 \%$ of multi-exonic mammalian genes undergo alternative splicing that leads to proteomic diversities [26]. Expression of different transcript variants and protein isoforms often occurs in cell type/tissue specific and developmental stage specific manner [27]. In the past decade, it was accumulated a lot of data about altering in splicing patterns of numerous genes (e.g. TP53, BCL2L1, TNR6, BARD1, AR, PI3K etc.) in cancer development and progression [28]. That is why, studying isoform expression profile is important for more comprehensive characterization of cancer heterogeneity and may reveal novel therapeutic targets. In current work, we showed that mRNA expression level of all CD150 isoforms in malignant B-cell lines did not exceed that in normal B cells or even was lower. Up to now, signaling properties of CD150 are associated with paired ITSM, which are present in MCD150, sCD150 and predicted CCD150 isoform. The primers that were used in our study discriminate between sCD150 isoform and mCD150 + cCD150 isoforms. The cCD150 isoform due to the lack of leader sequence could not be translocated to the endoplasmic reticulum for further translation and post-translation modifications. So, cCD150 translates into non-glycosylated protein core with molecular weight near $42 \mathrm{kDa}$. According to the western blot analysis of CD150 expression, we did not observe any band in the area of 40-50 kDa. This fact indirectly suggests that CCD150 is missing or expressed as a minor isoform in studied normal and malignant B cells and do not significantly affect the results obtained with primers to conventional cytoplasmic tail of CD150. Expression of SCD150 in HL cell lines, B-LCL and BL cell line Raji may indicate the role of secreted CD150 isoform in constitutive activation of CD150-mediated signaling pathways. Discovery of a novel CD150 isoform predominant expression in glial tumors raised the question whether expression level of $\mathrm{nCD} 150$ is higher than mCD150 in cells of immune system that could change conception of CD150 signaling properties. However, in our study, increased nCD150 expression level was revealed only in one studied cell line - pre-B-cell line NALM6, where CD150 protein was not detected. Taken together, the $\mathrm{mCD} 150$ with signaling properties is the main CD150 isoform in normal B-cell subsets, EBV-transformed B-LCLs and malignant B-cells lines. Moreover, we did not find any significant differences in quantitative ratio of mRNA expression level of studied CD150 splice isoforms between malignant B-cell lines and normal B-cell subtypes.

Mechanisms that underlie failure of CD150 protein expression during malignant transformation are cur- 
rently unknown. Multilevel regulation of gene expression embracing transcription, RNA processing, translation and post-translational level, allow cells strictly coordinate changes in profile of gene expression in response to internal and external stimuli [29]. The insufficiency of CD150 protein expression during malignant transformation of $B$ cells is probably associated with deviation at the post-transcriptional level of gene regulation. Post-transcriptional gene regulation includes processing of mRNA, export of mRNA to the cytoplasm and localization, mRNA stability and translation [30]. The key negative regulators of translation are short non-coding RNA - miRNAs. The miRNA can reduce target gene expression via induction of mRNA degradation or by inhibiting mRNA translation without affecting of mRNA level [31]. According to the miRNAs database "miRTarBase" (http://mirtarbase.mbc.nctu.edu.tw/ php/search.php?org=hsa\&kw=SLAMF1\&opt=target) near twenty miRNAs may potentially target CD150. However, no one putative miRNA for CD150 was validated by strong experimental approaches.

Translation initiation could depend on different ciselement present in 5'-UTR and/or 3'-UTR. Upstream open reading frames (UORF) in $5^{\prime}$-UTR - sequences franked by stop and start codons localised upstream of the main ORF, act as major regulator of translation initiation. Approximately $50 \%$ of human mRNAs contain UORF. The number of UORF in mRNA correlates with significantly reduced protein expression level [32]. Recently, it was shown that CD 150 has at least two isoforms based on their $5^{\prime}$-UTR structure [33]. Long isoform of SLAMF1 mRNA contains four UORF at $5^{\prime}$-UTR and is characterised by 5-6 times decreases its translation activity, in comparison with SLAMF1 mRNA short isoform without UORF in 5'-UTR [33]. It is possible that in malignant B-cell lines lacking expression of CD150 protein transcription may switch to the long mRNA isoforms leading to less efficient translation, as indicated by prevailing $m R N A$ over protein expression.

Taken together, malignant B-cell lines at the different stages of maturation only partially resemble their normal counterparts by CD150 expression. Mostly, neoplastic $B$ cells attempt to escape CD150 protein expression presumably using diverse mechanisms.

\section{ACKNOWLEDGEMENTS}

Authors thank to Prof. Eva Klein (Karolinska Institute, Stockholm, Sweden) and Prof. E.A. Clark (University of Washington, Seattle, WA, USA) for the cell lines, and Dr. M.Y. Yurchenko (Norwegian University of Science and Technology, Trondheim, Norway) for primers design and the help in the achievement of this study. The work was supported by scientific grant \#0113U008330 from the NAS of Ukraine and Scientific program of the NAS of Ukraine "Functional genomics and metabolomics in system biology" grant \#0112U0022194.

\section{REFERENCES}

1. Sidorenko SP, Clark EA. The dual-function CD150 receptor subfamily: the viral attraction. Nat Immunol 2003; 4: $19-24$.
2. Veillette A, Cruz-Munoz ME, Zhong MC. SLAM family receptors and SAP-related adaptors: matters arising. Trends Immunol 2006; 27: 228-34.

3. Cannons JL, Tangye SG, Schwartzberg PL. SLAM family receptors and SAP adaptors in immunity. Annu Rev Immunol 2011; 29: 665-705.

4. Sidorenko SP, Clark EA. Characterization of a cell surface glycoprotein IPO-3, expressed on activated human B and T lymphocytes. J Immunol 1993; 151: 4614-24.

5. Cocks BG, Chang CC, Carballido JM, et al. A novel receptor involved in T-cell activation. Nature 1995; 376: 260-3.

6. Punnonen J, Cocks BG, Carballido JM, et al. Soluble and membrane-bound forms of signaling lymphocytic activation molecule (SLAM) induce proliferation and Ig synthesis by activated human B lymphocytes. J Exp Med 1997; 185: 993-1004.

7. Bleharski JR, Niazi KR, Sieling PA, et al. Signaling lymphocytic activation molecule is expressed on CD40 ligandactivated dendritic cells and directly augments production of inflammatory cytokines. J Immunol 2001; 167: 3174-81.

8. De Salort J, Sintes J, Llinas L, et al. Expression of SLAM (CD150) cell-surface receptors on human B-cell subsets: from pro-B to plasma cells. Immunol Lett 2011; 134: 129-36.

9. Yurchenko MY, Kovalevska LM, Shlapatska LM, et al. CD150 regulates JNK1/2 activation in normal and Hodgkin's lymphoma B cells. Immunol Cell Biol 2010; 88: 565-74.

10. Rodriguez-Bayona B, Ramos-Amaya A, Brieva JA. Differential expression of SLAMS and other modulatory molecules by human plasma cells during normal maturation. Immunol Lett 2011; 134: 122-8.

11. Sidorenko SP, Vetrova EP, Yurchenko OV, et al. Monoclonal antibodies of IPO series against B cell differentiation antigens in leukemia and lymphoma immunophenotyping. Neoplasma 1992; 39: 3-9.

12. Mikhalap SV, Shlapatska LM, Yurchenko OV, et al. The adaptor protein $\mathrm{SH} 2 \mathrm{D} 1 \mathrm{~A}$ regulates signaling through CD150 (SLAM) in B cells. Blood 2004; 104: 4063-70.

13. Fanoni $D$, Tavecchio $S$, Recalcati $S$, et al. New monoclonal antibodies against B-cell antigens: possible new strategies for diagnosis of primary cutaneous B-cell lymphomas. Immunol Lett 2011; 134: 157-60.

14. Zucchetto A, Cattarossi I, Nanni $P$, et al. Cluster analysis of immunophenotypic data: the example of chronic lymphocytic leukemia. Immunol Lett 2011; 134: 137-44.

15. Bologna $\mathrm{C}$, Buonincontri $\mathrm{R}$, Serra $\mathrm{S}$, et al. SLAMF1 regulation of chemotaxis and autophagy determines CLL patient response. J Clin Invest 2016; 126: 181-94.

16. Aversa G, Chang CC, Carballido JM, et al. Engagement of the signaling lymphocytic activation molecule (SLAM) on activated T cells results in IL-2-independent, cyclosporin A-sensitive T cell proliferation and IFN-gamma production. J Immunol 1997; 158: 4036-44.

17. Romanets-Korbut O, Najakshin AM, Yurchenko M, et al. Expression of CD150 in tumors of the central nervous system: identification of a novel isoform. PLoS One 2015; 10: $\mathrm{e} 0118302$.

18. Isomaki P, Aversa G, Chang CC, et al. Expression of soluble human signaling lymphocytic activation molecule in vivo. J Allergy Clin Immunol 1999; 103: 114-8.

19. Kruse M, Meinl E, Henning G, et al. Signaling lymphocytic activation molecule is expressed on mature CD83+ dendritic cells and is up-regulated by IL-1 beta. J Immunol 2001; 167: 1989-95.

20. Yurchenko MY, Kashuba EV, Shlapatska LM, et al. The role of CD150-SH2D1A association in CD150 signaling in Hodgkin's lymphoma cell lines. Exp Oncol 2005; 27: 24-30. 
21. Shlapatska LM, Kovalevska LM, Gordiienko IM, et al. Intrinsic defect in B-lymphoblastoid cell lines from patients with X-linked lymphoproliferative disease type 1. II. Receptormediated Akt/PKB and ERK1/2 activation and transcription factors expression profile. Exp Oncol 2014; 36: 162-9.

22. Shaffer AL, Young RM, Staudt LM. Pathogenesis of human B cell lymphomas. Annu Rev Immunol 2012; 30: 565-610.

23. Basso K, Dalla-Favera R. Germinal centres and B cell lymphomagenesis. Nat Rev Immunol 2015; 15: 172-84.

24. Kuehl WM, Bergsagel PL. Molecular pathogenesis of multiple myeloma and its premalignant precursor. J Clin Invest 2012; 122: 3456-63.

25. Takeda S, Kanbayashi D, Kurata T, et al. Enhanced susceptibility of B lymphoma cells to measles virus by Epstein - Barr virus type III latency that upregulates CD150/ signaling lymphocytic activation molecule. Cancer Sci 2014; 105: $211-8$.

26. Chen J, Weiss WA. Alternative splicing in cancer: implications for biology and therapy. Oncogene 2015; 34: 1-14.

27. Zhang Z, Pal S, Bi Y, et al. Isoform level expression profiles provide better cancer signatures than gene level expression profiles. Genome Med 2013; 5: 33.

28. Oltean S, Bates DO. Hallmarks of alternative splicing in cancer. Oncogene 2014; 33: 5311-8.
29. Carpenter S, Ricci EP, Mercier BC, et al. Post-transcriptional regulation of gene expression in innate immunity. Nat Rev Immunol 2014; 14: 361-76.

30. Jackson RJ, Hellen CU, Pestova TV. The mechanism of eukaryotic translation initiation and principles of its regulation. Nat Rev Mol Cell Biol 2010; 11: 113-27.

31. Valinezhad Orang A, Safaralizadeh R, KazemzadehBavili M. Mechanisms of miRNA-mediated gene regulation from common downregulation to mRNA-specific upregulation. Int J Genomics 2014; 2014: 970607.

32. Barbosa C, Peixeiro I, Romao L. Gene expression regulation by upstream open reading frames and human disease. PLoS Genet 2013; 9: e1003529.

33. Putlyaeva LV, Schwartz AM, Korneev KV, et al. Upstream open reading frames regulate translation of the long isoform of SLAMF1 mRNA that encodes costimulatory receptor CD150. Biochemistry (Mosc) 2014; 79: 1405-11.

34. Shlapatska LM, Kovalevska LM, Gordiienko IM, et al. Intrinsic defect in B-lymphoblastoid cell lines from patients with X-linked lymphoproliferative disease type 1. I. Cell surface phenotype and functional studies. Exp Oncol 2014; 36: 2-8.

35. Nagy N, Cerboni C, Mattsson K, et al. SH2D1A and SLAM protein expression in human lymphocytes and derived cell lines. Int J Cancer 2000; 88: 439-47. 